\title{
A magneto-gravitational trap for studies of gravitational quantum states
}

\author{
V. V. Nesvizhevsky ${ }^{1, a}$, F. Nez ${ }^{2}$, S. A. Vasiliev ${ }^{3}$, E. Widmann ${ }^{4}$, P. Crivelli ${ }^{5}$, S. Reynaud ${ }^{2}$, A. Yu. Voronin ${ }^{6}$ \\ ${ }^{1}$ Institut Max von Laue, Paul Langevin, 71 avenue des Martyrs, 38042 Grenoble, France \\ ${ }^{2}$ Laboratoire Kastler Brossel, Sorbonne Université, CNRS, ENS-PSL Université, Collège de France, 75252 Paris, France \\ ${ }^{3}$ Department of Physics and Astronomy, University of Turku, FI-20014 Turku, Finland \\ ${ }^{4}$ Stefan Meyer Institute for Subatomic Physics, Austrian Academy of Sciences, Boltzmanngasse 3, 1090 Wien, Austria \\ ${ }^{5}$ ETH, Zurich, Institute for Particle Physics and Astrophysics, 8093 Zurich, Switzerland \\ ${ }^{6}$ Lebedev Institute, 53 Leninsky pr., Moscow 119333, Russia
}

Received: 23 January 2020 / Accepted: 27 May 2020 / Published online: 10 June 2020

(C) The Author(s) 2020

\begin{abstract}
Observation time is the key parameter for improving the precision of measurements of gravitational quantum states of particles levitating above a reflecting surface. We propose a new method of long confinement in such states of atoms, anti-atoms, neutrons and other particles possessing a magnetic moment. The earth gravitational field and a reflecting mirror confine particles in the vertical direction. The magnetic field originating from electric current passing through a vertical wire confines particles in the radial direction. Under appropriate conditions, motions along these two directions are decoupled to a high degree. We estimate characteristic parameters of the problem, and list possible systematic effects that limit storage times due to the coupling of the two motions.
\end{abstract}

\section{Introduction}

Gravitational quantum states (GQSs) of light neutral particles (hydrogen $(H)$, deuterium $(D)$, helium $(H e)$, anti-hydrogen $(\bar{H})$ atoms, neutrons $(n)$ and others) levitating above a reflecting surface can be used to improve the precision of various measurements. The observation of GQSs of $n$ [1-7] initiated active analysis of the peculiarities of this phenomenon [8-15] and its applications to the searches for new physics, such as the searches for extra fundamental short-range interactions [16-22], verification of weak equivalence principle in the quantum regime [23-25], extensions of quantum mechanics $[26,27]$, extensions of gravity and space theories [28-31], tests of Lorentz invariance [32-34] and others. Spectroscopy and interferometry methods of observation of GQSs of $n$ have been analyzed theoretically and implemented experimentally

a e-mail: nesvizhevsky@ill.eu (corresponding author) over the previous two decades [35-39]. A $n$ whisperinggallery phenomenon in which acceleration replaces gravity was observed and studied [40]. The idea to measure GQSs of $\bar{H}$ was proposed and developed in a series of theoretical publications [41-46]. The same approach can be readily extended to $H$ atoms, and the work on the development of a high-density $H$ source has started [47]. Other particles, like positronium, have been discussed [48].

Observation time is the key parameter that controls the precision. We propose a new method of long confinement of neutral particles possessing a magnetic moment in GQSs in a Magneto-Gravitational Trap (MGT). A key feature of the new method is the combination of the vertical confinement by gravity and quantum reflection from a mirror and the radial confinement by the magnetic field of a vertical linear current. Both confinement principles are well established [3,49-53]. However, the combination of the two approaches seems to be challenging as the magnetic field might produce large false effects thus making impossible any precision studies of GQSs. We show that one can achieve small mixing of radial and vertical motions and thus can control false effects to an acceptable degree.

The most typical trap for cold atoms is the MagnetoOptical Trap (MOT) [54]. It combines magnetic trapping and optical cooling. Such a trap was used in the groundbreaking experiments on Bose-Einstein Condensate (BEC) in a gas of ultracold atoms [55,56]. Since there is no maximum magnetic field in 3D, low-field-seeking (lfs) atoms are trapped in MOTs in the field minimum. Since they are not in the lowest internal state, any disturbance (collisions of atoms, magnetic field inhomogeneities) can flip their spin. This magnetic relaxation to the untrapped state is the main mechanism of losses from MOTs. However, it is absent for 
traps for high-field-seeking (hfs) atoms, which may therefore allow the trapping of clouds of atoms of much higher density. Dynamic magnetic traps for hfs atoms based on rapidly varying electromagnetic fields have been proposed [79] but they are typically shallow.

In contrast, the MGT provides a deep trapping potential and is especially suitable for the lightest atoms: $H$ and $D$. For $H$, a trap barrier height of $\sim 0.5 \mathrm{~K}$ can be easily realized, which allows trapping of a large number of atoms at temperatures of $\sim 100 \mathrm{mK}$. Optical cooling methods based on the $1 S-2 P$ or two-photon $1 S-2 S$ transitions can be used down to the recoil limit of $\sim 2 \mathrm{mK}$. Further cooling of the trapped gas can be done using evaporation over the trap barrier. Atomic collisions in the high-density regime provide high equilibration rate leading to substantially lower temperatures.

Since the general principles of operation of the MGT are the same for different particles, we first describe them in a general way in Sect. 2, and coupling of vertical and radial motions of the particles in the MGT is analyzed in Sect. 3. However, a specific implementation of the MGT and experimental methods (type of mirror, electric current, particle loading/unloading and storage time, size, temperature, specific interferometry and spectroscopy method, etc) depends on the type of particle (neutron, atom, antiatom, etc) as well as their velocity spectrum. In specific examples, we will indicate the reason why the respective method was chosen. The feasibility of loading/unloading the MGT as well as examples of precision measurements of GQSs in the MGT are presented in Sect. 4.

We focus on the properties of the MGT and leave the crucial topics of specific implementations of loading/unloading the trap and spectroscopy/ interferometry of GQSs for later publications. We will only show the feasibility of their implementation and their compatibility with the operation of the MGT. Note that general methods of spectroscopy/interferometry of GQSs have been developed in detail $[40,43,46,57,59]$, and that fast changes of the electric current can be used to load/unload the MGT in case of atoms and anti-atoms, while super-fluid ${ }^{4} \mathrm{He}$ in the trap exposed to the flux of cold neutrons allows producing ultra-cold neutrons (UCNs) directly in the trap [65].

\section{Description of the trap}

Figure 1 shows a scheme of the MGT. The mirror and gravitational field confine particles vertically. The interaction of a particle's magnetic moment with the vertical electric current and the centrifugal acceleration confine particles radially.

Under appropriate conditions that will be discussed in the paper, in particular for a sufficiently long wire, forces

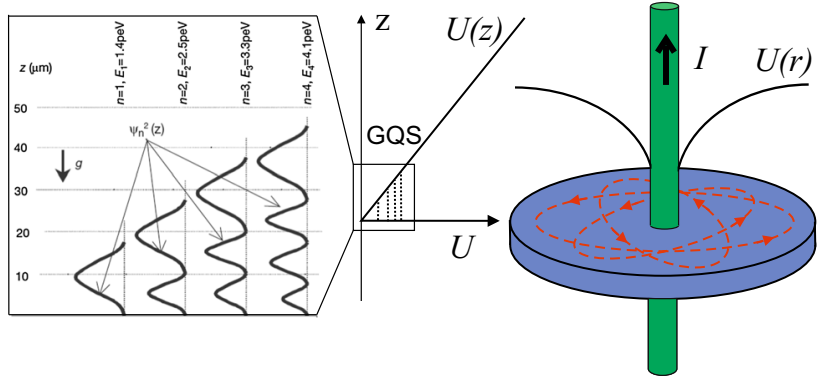

Fig. 1 On the right side, a schematic representation of the MagnetoGravitational trap (MGT) for neutral particles with a magnetic moment is shown. The linear gravity potential and quantum reflection from the horizontal mirror (blue) confine particles in the vertical direction. The particle's wave functions in four lowest GQSs are shown in the insert on the left as a function of the height above the mirror. The magnetic field of a vertical linear wire carrying an electric current $I$ and the centrifugal acceleration confine particles in the horizontal plane. The magnetic field is inversely proportional to the distance to the wire. The particle adiabatically moves along closed elliptical trajectories (red dashed lines around the wire), similar to the orbital motion of planets around the sun

acting on particles in the vertical and horizontal directions are almost orthogonal so that the corresponding motions are decoupled to a high degree. The vertical motion of particles with lowest vertical energies is governed by quantum mechanics while the horizontal motion can be considered classical in realistic conditions.

\subsection{Motion of a magnetic dipole in the field of a linear current}

The quantum motion of a magnetic dipole in the magnetic field of linear current can be described analytically $[66,67]$. Here, an adiabatic approximation, which allows classical treatment, is sufficient for our purpose. It relies on the hierarchy of characteristic times associated with the fast spin motion and the slowly varying magnetic field in a frame comoving with the particle in the plane perpendicular to the current. In the MGT, the particle trajectories follow Keplerlike orbits around the wire. For the simplest case of a circular orbit with radius $r$, the rotation frequency $\Omega$ of a magnetic dipole $\mu$ is given by:

$\Omega^{2}=\frac{\mu_{0} \mu I}{2 \pi r^{3} m}=\frac{B(r) \mu}{r^{2} m}$,

where $I$ is the electric current, $\mu_{0}$ the magnetic permeability of vacuum, $m$ the particle mass, and $r$ the radial distance from a given point to the wire carrying the current. To meet the adiabaticity condition, the angular velocity of rotation has to be much smaller than the Larmor frequency $\omega_{L}$ of the spin precession, $\Omega \ll \omega_{L}=\gamma B(r)$, with $\gamma$ being the gyromagnetic ratio for the magnetic dipole under consideration. 
Radial confinement will occur in the Coulomb-like potential:

$U(r)=-\mu B(r)=-\frac{\mu_{0} \mu I}{2 \pi r}$,

with the Bohr energy and the Bohr radius of the particle

$E_{B}=\frac{m}{2 \hbar^{2}}\left(\frac{\mu_{0} \mu I}{2 \pi}\right)^{2}, R_{B}=\frac{2 \pi \hbar^{2}}{m \mu_{0} \mu I}$.

The Coulomb potential depth reaches a maximum at the wire surface. The value of the potential strength depends on the type of particle; it is roughly three orders of magnitude larger for electron spins than for nuclear spins, or for the neutron spin. Therefore, one needs much stronger magnetic fields and electric currents for trapping $n$ or ${ }^{3} \mathrm{He}$.

Let's evaluate the feasibility of achieving magnetic trapping for $n$ with their small magnetic moments. The current density in the wire is $i_{0}=I / \pi R^{2}$, where the wire radius $R$ is a free parameter. A typical value for the most common superconductors based on NbTi is $i_{0} \approx 10^{5} \mathrm{~A} / \mathrm{cm}^{2}$ in a magnetic field of $3 \mathrm{~T}$ and a temperature of $4.2 \mathrm{~K}$. With this current density constraint, the potential depth as a function of the wire radius is

$U(R)=\frac{1}{2} \mu_{0} \mu i_{0} R$.

It looks that increasing the wire radius is beneficial for making a stronger magnetic trap. This is, however, only true until we reach another constraint associated with the maximum critical current density. Then the current density has to be reduced. This effect depends on the type and manufacture of the wire and the temperature. For the values given above and a wire radius of $0.5 \mathrm{~cm}$, the field near the wire surface is $B_{\max } \sim 3 \mathrm{~T}$, which is close to the critical value. Therefore, the specified maximum field and the trap depth are reached at a wire radius of $0.5 \mathrm{~cm}$; further increase of the wire radius would not help. The field strength of $3 \mathrm{~T}$ corresponds to the trap depth of $\sim 2 \mathrm{mK} \sim 1.5 \times 10^{-7} \mathrm{eV}$ for $n$, values typical for ultra-cold neutrons $[70,71]$. Further improvement can be obtained by a decrease of temperature of the wire to $1.5-1.7$ $\mathrm{K}$ or the use of a superconductive wire with a larger ratio of $\mathrm{NbTi} / \mathrm{Cu}$. This may increase the trap depth by a factor of $2-3$, thus making magnetic trapping of the full UCN energy range quite realistic.

Using the MGT for atoms with unpaired electron spin (H and D) in a high field seeking state should be much easier because of their much larger magnetic moments. We can reduce the current by three orders of magnitude, or, keeping the same current density, decrease the wire radius, or trap atoms at higher temperatures. Reducing the wire radius, one should take care of not violating the adiabaticity condition for the atomic motion at the smallest distances to its surface
Table 1 Typical parameters (orbit radius $r$, energy $E$, rotation frequency $\Omega$, velocity $v$ ) of a particle (hydrogen atom, deuterium atom, neutron) state bound in the magnetic field of a linear current $I$

\begin{tabular}{llllll}
\hline Particle & $I(\mathrm{~A})$ & $r(\mathrm{~cm})$ & $E(\mathrm{eV})$ & $\Omega /(2 \pi)(\mathrm{Hz})$ & $v(\mathrm{~m} / \mathrm{s})$ \\
\hline $\mathrm{H}$ & 10 & 1 & $5.810^{-9}$ & 16.5 & 1.0 \\
$\mathrm{H}$ & 10 & 0.5 & $1.210^{-8}$ & 47.4 & 1.5 \\
$\mathrm{D}$ & 10 & 1 & $5.810^{-9}$ & 11.8 & 0.74 \\
$\mathrm{D}$ & 10 & 0.5 & $1.210^{-8}$ & 33.5 & 1.1 \\
$\mathrm{n}$ & 10000 & 1 & $5.810^{-9}$ & 16.5 & 1.0 \\
$\mathrm{n}$ & 10000 & 0.5 & $1.210^{-8}$ & 47.4 & 1.5 \\
\hline
\end{tabular}

Table 2 Eigenvalues $\lambda_{i}$ (roots of Airy function), gravitational energies $E_{i}$, characteristic transition frequencies $v_{i}$ and classical turning points $z_{i}$ for neutrons, hydrogen and anti-hydrogen atoms in the Earth's gravitational field above a mirror. Index ${ }_{i}$ stands for the quantum state number

\begin{tabular}{lllcl}
\hline$i$ & $\lambda_{i}$ & $E_{i}(\mathrm{peV})$ & $\nu_{i}(\mathrm{~Hz})$ & $z_{i}(\mu m)$ \\
\hline 1 & 2.338 & 1.407 & 340.1 & 13.73 \\
2 & 4.088 & 2.461 & 594.8 & 24.00 \\
3 & 5.521 & 3.324 & 803.7 & 32.41 \\
4 & 6.787 & 4.086 & 988.0 & 39.85 \\
5 & 7.944 & 4.782 & 1156.3 & 46.64 \\
6 & 9.023 & 5.431 & 1313.2 & 52.97 \\
7 & 10.04 & 6.044 & 1461.4 & 58.94 \\
\hline
\end{tabular}

when $r \approx R$. Keeping the same constraint of the fixed current density, the Larmor precession frequency scales as $\omega_{L} \sim r$, while the orbital rotation frequency scales as $\Omega \sim 1 / \sqrt{r}$. However, even having a micrometer radius wire still does not violate adiabaticity, and such a trap could be realized for $\mathrm{H}$ and D.

Table 1 presents typical parameters of various particles in the MGT.

\subsection{Gravitational quantum states}

The particle is confined vertically by the gravitational field and a mirror. This motion is quantized and described by GQSs. Such states were predicted [1] and discovered [3] for $n$, and predicted for $\bar{H}$ and $H$ atoms [41]. All details about the physical properties of such states can be found in the cited papers; here, we give only a summary of the main properties of these states in Table 2 for the reader's convenience.

The characteristic energy, spatial and time scales of such states are given by:

$\varepsilon_{g}=\sqrt[3]{\hbar^{2} m g^{2} / 2}, l_{g}=\sqrt[3]{\hbar^{2} /\left(2 m^{2} g\right)}, \tau_{g}=\frac{4 m l_{g}^{2}}{\sqrt{2 \pi} \hbar}$. 
The surface of the mirror should be flat enough, and the roughness should be small enough to provide specular reflection of the particles. The material of the mirror for $n$ must have a positive neutron-optical potential and a low loss coefficient. Neutron-optical potential arises due to the coherent interaction of the neutron with nuclei in matter and was introduced by Enrico Fermi in [80]. Most materials satisfy these conditions. Quantum reflection of (anti)atoms from the surface can be provided by their interaction with the van der Waals/Casimir-Polder potential of the surface. The mirror materials for (anti)atoms is chosen so as to increase the probability of quantum reflection and/or provide high control of this interaction. Since the quantum reflection of (anti)atoms occurs without their direct contact with the surface, the requirements for the mirror material are the same for atoms and antiatoms. High reflection is provided by the surface of liquid $\mathrm{He}$, and this process is studied for instance in Ref. [68].

\section{Coupling of vertical and radial motions}

Vertical and horizontal motions of particles are decoupled only to a finite precision. Below we consider phenomena which can mix them.

\subsection{The effect of wire non-verticality and mirror non-horizontality}

Although the precision of setting the mirror and wire directions can be high, the magnetic field would slightly deviate from Eq. (2), in particular due to the environment. A vertical field gradient could result in false effects. To estimate these, we derive an expression for the magnetic field in the vicinity of a particle's circular trajectory.

$B=\frac{\mu_{0} I}{2 \pi \rho}$

$\rho=\sqrt{(r \cos (\varphi) \cos (\alpha)-z \sin (\alpha))^{2}+r^{2} \sin ^{2}(\varphi)}$.

Here $\rho$ is the distance between the wire and a particle, $\varphi$ is the particle angle in the horizontal plane, $z$ is the vertical coordinate of a particle above the mirror plane, $\alpha$ is the angle between the wire and vertical direction (in $\mathrm{x}-\mathrm{z}$ plane).

Below, we show that the ratio $z / r \ll 1$ is small for all states of interest. Taking into account the smallness of the deviation of $\alpha$ from the vertical direction, the potential energy is:

$U(z) \simeq-\frac{\mu_{0} \mu I}{2 \pi r}\left(1+\frac{z \alpha \cos (\varphi)}{r}\right)$.
Table 3 Extra acceleration and the gravitation transition frequency shift in the magnetic field of a linear current due to the non-verticality of wire alignment $\left(\hbar \Delta \omega_{21}=\Delta E_{2}-\Delta E_{1}\right)$

\begin{tabular}{lrllll}
\hline Particle & $I(\mathrm{~A})$ & $\alpha$ & $r(\mathrm{~cm})$ & $a_{0}\left(\mathrm{~m} / \mathrm{s}^{2}\right)$ & $\Delta \omega_{21}(\mathrm{~Hz})$ \\
\hline $\mathrm{H}$ & 10 & $10^{-3}$ & 1 & 0.055 & -0.003 \\
$\mathrm{H}$ & 10 & $10^{-4}$ & 1 & 0.0055 & -0.00003 \\
$\mathrm{H}$ & 10 & $10^{-3}$ & 0.5 & 0.22 & -0.05 \\
$\mathrm{n}$ & 10000 & $10^{-3}$ & 1 & 0.0055 & -0.003 \\
$\mathrm{n}$ & 10000 & $10^{-4}$ & 1 & 0.055 & -0.00003 \\
$\mathrm{n}$ & 10000 & $10^{-3}$ & 0.5 & 0.22 & -0.05 \\
\hline
\end{tabular}

The corresponding vertical component of acceleration due to the wire non-verticality is:

$a_{m}=a_{0} \cos (\varphi), a_{0}=\frac{\mu_{0} \mu I}{2 \pi m r^{2}} \alpha$.

Due to the periodicity of the function $\cos (\varphi(t))$, a gravitational energy correction due to the extra acceleration in the gradient magnetic field vanishes in the first order of the small parameter $a_{0} / g$ :

$\varepsilon_{g}^{\prime}=\frac{1}{2 \pi} \int\left(\frac{m \hbar^{2}\left(g+a_{0} \cos (\varphi)\right)^{2}}{2}\right)^{1 / 3} d \varphi$.

The first non-vanishing correction to the unperturbed gravitation energy level $E_{i}=m g z_{i}$ appears in the second order:

$\Delta E_{i}=-\frac{1}{18} \frac{m z_{i} a_{0}^{2}}{g}$.

In Table 3, we present typical values of $a_{0}$ and the correction to the frequency shift between second and first GQSs for different orbits of a trapped particle.

As one can see in Table 1, the particle rotation frequencies on orbits in the trap are small compared to the transition frequencies between low GQSs. Thus, no resonance effects could be found.

An even smaller systematic effect is associated with the broadening of the peak of the resonance transitions between GQSs. It would be caused by transitions between GQSs due to the non-verticality of the wire, which would decrease storage times in GQSs. An additional suppression factor arises from the fact that under the adiabaticity condition, which is valid for all practically interesting cases, there are no transitions between GQSs caused by the wire non-verticality. 
3.2 Effect of a non-vanishing vertical gradient of the magnetic field

A non-vanishing vertical gradient of the magnetic field could be due to a finite trap size. It might produce sizable effects which have to be compensated to a maximum degree. A residual gradient would result in a transition frequency shift proportional to the current. Thus, it could be extracted from experimental data by extrapolating the frequency shift to zero current.

\subsection{Effect of vibration of the wire and mirror}

The wire vibration effect can be estimated by assuming that the angle between the wire and the vertical direction is a periodic function of time, which is changing with an oscillation frequency:

$\alpha(t)=\alpha_{0} \cos \left(\omega_{v} t\right)$

Then the perturbing potential is:

$U(z, t) \simeq \frac{\mu_{0} \mu I}{2 \pi r} \frac{z \alpha_{0} \cos \left(\omega_{v} t\right) \cos (\varphi)}{r}$.

Though its amplitude is small, as established above, oscillations of the perturbing potential could appear to be in resonance with the transition frequency between GQSs. In this case, the transition probability $P_{i k}$ between initial $(i)$ and final ( $k$ ) GQSs is given by the Rabi-type expression [69]:

$P_{i k}=\sin ^{2}\left(\Omega_{i k} t\right)$

Here, the transition rate is:

$\Omega_{i k}=\frac{\mu_{0} \mu I}{4 \pi r} \frac{\alpha_{0} l_{g}^{3}}{\left|z_{i}-z_{k}\right|^{2}}$.

The time $T_{i k}$ needed for the complete transition from state $i$ to state $k$ is

$T_{i k}=\frac{\pi}{2 \Omega_{i k}}$

Characteristic times $T_{12}$ are given in Table 4 .

A similar effect of parasitic transitions between GQSs caused by vibrations of the mirror was studied theoretically and experimentally in Ref. [64]. The main conclusion of this work, as well as the estimations in this paper, is that one has to design the experimental setup in such a way as to suppress the vibrations of its components with frequencies close to the frequencies of the resonant transitions between GQSs. Moreover, one should measure the vibration spectrum of the
Table 4 Transition times from the first to the second gravitational state caused by the oscillation of the wire and estimated in accordance with Eq. (16)

\begin{tabular}{lrllr}
\hline Particle & $I(\mathrm{~A})$ & $\alpha_{0}$ & $r(\mathrm{~cm})$ & $T_{12}(\mathrm{~s})$ \\
\hline $\mathrm{H}$ & 10 & $10^{-3}$ & 1 & 93.2 \\
$\mathrm{H}$ & 10 & $10^{-4}$ & 1 & 931.9 \\
$\mathrm{H}$ & 10 & $10^{-3}$ & 0.5 & 46.6 \\
$\mathrm{n}$ & 10000 & $10^{-3}$ & 1 & 93.2 \\
$\mathrm{n}$ & 10000 & $10^{-4}$ & 1 & 931.9 \\
$\mathrm{n}$ & 10000 & $10^{-3}$ & 0.5 & 46.6 \\
\hline
\end{tabular}

wire and the mirror and make sure that there are no dangerous frequencies in the spectrum. Otherwise, the probability of parasitic transitions can be significant.

\subsection{Effect of Earth's rotation}

The effect of Earth's rotation results in an additional Coriolis acceleration that a moving particle acquires in the noninertial frame. The vertical component of such an acceleration $a_{c}$ of a particle trapped on a circular orbit is given by:

$a_{c}=\Omega_{E} \cos (\Theta) \sqrt{\frac{\mu_{0} \mu I}{\pi m r}} \cos (\varphi)$.

Here, $\Omega_{E}=7.27 \times 10^{-5} \mathrm{rad} / \mathrm{s}$ is the Earth's rotation frequency around its axis, $\Theta$ is a latitude of geographic position ( $45^{\circ}$ in Grenoble). In case of an $H$ atom trapped in a circular orbit with radius $r=0.5 \mathrm{~cm}$ and current $I=10 \mathrm{~A}$, the acceleration is $a_{c}=7.6 \times 10^{-5} \mathrm{~m} / \mathrm{s}^{2}$.

After averaging over the trajectory, the first order Coriolis effect is canceled due to the periodic $\cos (\varphi)$ factor. The second order Coriolis effect is well below the accuracy of our experiment and can be neglected.

\section{Feasibility of precision studies of GQS in the MGT}

The goal of this chapter is to show the principle feasibility of precision studies of GQSs in the MGT. Concrete measuring schemes would be developed in other papers. The prove of feasibility can be achieved by fulfilling the following conditions: (a) long storage of neutral particles in GQSs, (b) the ability to load and unload the trap, (c) the possibility of spectroscopy and interferometry of particles in the MGT.

By long storage times of particles in GQSs we mean, in this context, the times much longer than the characteristic time of formation of GQSs as defined in Eq. (5) that is equal to $\sim 1 \mathrm{~ms}$. In this paper, we limit the analysis of storage times to only systematic effects associated with the MGT, namely, 
all effects mixing horizontal and vertical motion of particles in the MGT. They are presented in Sect. 3. The other systematic effects are not specific to the presented method of storing particles in the MGT and are analyzed in detail in our previous works, as well as in works of other groups working in this field. Among those systematic effects can be noted the frequency shifts associated with the van der Waals/ CasimirPolder interaction [58] or with the excitation of resonant transitions [59,60], finite resolution of GQSs associated with the effect of absorber/scatterer [6,61,62], quenching of GQSs by surface charges [63], parasitic transitions between GQSs induced by vibrations of the mirror [64], various effects associated with waviness and roughness of the mirror surface, parasitic magnetic fields, thermal effects and so on.

Convenient options for loading and unloading the MGT are presented in Sect. 4.1. They are different for $H / \bar{H}$ and $n$ due to the large difference of their magnetic moments and the methods of their production.

In Sect. 4.2, we extend the method of spectroscopy of GQSs to $H$ atoms: it is compatible with the MGT operation.

In Sect. 4.3, we propose a method of interferometry with GQSs of $\bar{H}$ compatible to the MGT operation.

\subsection{Loading/unloading the MGT}

The MGT can be loaded with $H, \bar{H}$, for instance, by rapidly switching the wire current. This is technically feasible due to the relatively large magnetic moment (compared to the neutron magnetic moment), thus, the relatively small electric currents needed to trap sufficiently slow $H, \bar{H}$. For an atom velocity of $\sim 1 \mathrm{~m} / \mathrm{s}$ and a trap size of $\sim 10^{-2} \mathrm{~m}$, the $\sim$ 10 A current switching time should be significantly shorter than $\sim 10 \mathrm{~ms}$ (see Table 1). The trap phase-space volume is estimated by assuming that all atoms with a velocity $v$ lower than the escape velocity $v_{c}$ for a given atom-wire distance would be captured. Then, the number of captured atoms is:

$N=2 f_{0} \sqrt{\frac{\mu_{0} \mu I}{\pi m}}\left(\sqrt{r_{2}}-\sqrt{r_{1}}\right)$.

Here, $r_{2}, r_{1}$ are maximum and minimum radius of the trap, $f_{0}$ is the average density of atoms in a phase volume which is characterized by the maximum velocity $v_{\max }=$ $\sqrt{\mu_{0} \mu I /\left(\pi m r_{1}\right)}$ and the spatial size $2 \pi\left(r_{2}^{2}-r_{1}^{2}\right)$.

In the case of $n$, rapid switching of the wire current is not feasible because of very large current values. In contrast, $n$ offer another particularly elegant method of loading the MGT. It consists of producing UCNs directly in the trap filled in with superfluid ${ }^{4} \mathrm{He}$ [65]. This method provides the highest phase-space densities of $>10^{3} \mathrm{n} / \mathrm{cm}^{3}$ and allows avoiding UCN losses associated with their extraction and transportation. A few dozen small mirrors can be superimposed in one experimental setup (within the limits of the height of a cold neutron beam). For the typical parameters of the existing intense cold neutron beams [76,77], a conservative estimation of the number of UCNs that can be trapped simultaneously in one GQS is $10^{-2}-10^{-1}$, which roughly corresponds to count rates in the existing GQS experiments. The main difference of the proposed method is that it can provide a much longer time of observation of $n$ GQSs, comparable with the neutron lifetime.

Other methods include adiabatically changing the wire current or an additional uniform magnetic field in certain mirror geometries, spin-flip by a radio-frequency magnetic field, and various mechanical devices.

\subsection{Resonance spectroscopy}

Methods of spectroscopy of GQSs [35] have been developed in detail theoretically and experimentally. In the case of $n$, it was realized by the qBounce collaboration using excitation by mechanical vibrations of the bottom mirror $[37,38,78]$. Non-resonant transitions to a set of GQSs were used by the Tokyo collaboration [39]. GRANIT currently measures resonant transitions induced by a periodically changing magnetic field gradient $[57,59]$. For $\bar{H}$, resonant spectroscopy of GQSs was discussed in [59]. The theoretical formalism and experimental methods can be easily extended to $H$. Taking into account the large magnetic moment of the $H$ atoms and the need to measure at cryogenic temperatures, we consider the method of magnetic excitation of resonant transitions between GQSs of $H$ atoms to be most appropriate.

Due to the large spatial extension of GQSs and the magnetic moment of $H$, one can observe in the MGT the resonant changes in spatial density of the particles localized in GQSs above a mirror as a function of the oscillating frequency of an additional vertical magnetic field gradient. The resonant transitions result from the interaction of the magnetic dipoles of the trapped particles with the field gradient. The changes in spatial density are enhanced when the oscillating frequency coincides with the transition frequency $\omega_{i k}=\left(E_{k}-E_{i}\right) / \hbar$. The additional field is assumed to have the form:

$\mathbf{B}(z, t)=B_{0} \mathbf{e}_{z}+\beta \cos (\omega t)\left(z \mathbf{e}_{z}\right)$.

Here, $B_{0}$ is the amplitude of the static field component, $\beta$ is the oscillating magnetic field gradient; this equation is valid for $z$ values much smaller that the size of the magnetic system. The oscillation frequency is determined by the transition frequency between the lowest GQSs; its typical value is $\omega \sim 10^{3}$ $\mathrm{rad} / \mathrm{s}$. For simplicity we omitted any radial components of the magnetic field, which do not influence the dynamics in the system. The static axial component $B_{0}$ provides a nonzero $z$ component of the atomic magnetic moment inducing the force in the oscillating field gradient. The strength of this component should not exceed the characteristic strength 
of the trapping field. Such a field configuration can be provided with a pair of coils in the anti-Helmholtz configuration arranged around the trap.

First, $H$ are prepared in the ground GQS. This is achieved by absorbing highly excited states using a scatterer/absorber plate lowered down to a certain height $H_{a}$ above the mirror surface. Adjusting the $H_{a}$ value close to the characteristic delocalization height of the ground state $l_{0} \lambda_{1}<H_{a}$ (Eq. 5) will effectively remove all other GQSs from the trap. This technique was used for in-beam spectroscopy of GQSs of $n$ $[6,43,57,74]$. Vertical motion of the absorber over $\sim 10 \mu \mathrm{m}$ can be achieved with piezo-actuators.

At the second stage, the absorber is lifted up by $20-30$ $\mu \mathrm{m}$ to allow trapping a few exited GQSs. An oscillating vertical gradient of the magnetic field is applied at a frequency $\omega$. The field induces transitions from the ground to excited GQSs with a probability, which depends resonantly on the oscillating frequency $\omega_{1 k}=\left(E_{k}-E_{1}\right) / \hbar$.

Third, the number of $H$ remaining in the ground GQS can be measured by placing the absorber down again and eliminating $H$ in excited states. The numbers of $H$ in the ground state before the excitation and after can be measured by releasing them from the trap to a detector.

The probability to excite $H$, initially prepared in the ground state, is given by the following expression:

$$
\begin{aligned}
P(t) & =\left|\sum_{k} C_{k}(t) \exp \left(-i E_{k} t / \hbar\right) \int_{H_{d}}^{\infty} \chi_{k}(x) d x\right|^{2} . \\
\chi_{k}(x) & =\frac{\operatorname{Ai}\left(x-\lambda_{k}\right)}{\operatorname{Ai}^{\prime}\left(-\lambda_{k}\right)} .
\end{aligned}
$$

Here, $t$ is the time of interaction of $H$ in the ground state with the oscillating magnetic field gradient, and $C_{k}(t)$ is the population of quantum state $k$ at time $t$. Assuming that the field frequency is close to the resonance $\omega_{1 k}$ and the time is sufficiently large, $t \gg \hbar / \omega_{1 k}$, one can get a simplified expression for the probability averaged over a period $\hbar / \omega_{1 k}$ :

$\bar{P}(t)=\exp (-\Gamma t / \hbar) \sum_{k}\left|C_{k}(t)\right|^{2}\left|\int_{H_{d}}^{\infty} \chi_{k}(x) d x\right|^{2}$,

with $\Gamma=\omega_{g} \operatorname{Im} \lambda_{i}$ the width of a quasi-stationary state.

The resonance frequency value corresponds to a maximum loss of $H$ from the ground state as a function of the applied magnetic field frequency. Similarly, it is possible to measure transitions between any other pairs of low GQSs.

\subsection{Interferometry of quasi-stationary states}

Interferometric methods of observation of GQSs are based on the use of position-sensitive or time-resolving detectors. Such detectors are routinely used for $n$, and respective exper- imental methods have been developed in detail in studies of neutron whispering gallery [40]; we do not reproduce these details here. For $H$, efficient detectors of this type still have to be developed; therefore, we cannot yet propose a reliable experimental scheme for $H$. Here, we propose a new interferometric method of measurement of simultaneously several GQSs of $\bar{H}$. It combines the efficient use of $\bar{H}$ produced, easy implementation and high precision. The method is based on the observation of a time distribution of detection events at a given $z$-location, or a vertical position distribution of detection events at a given time. An interference pattern can be observed if a pure initial state or a superposition of states is shaped.

Here, we analyze an example of the time evolution of an initially prepared wave-packet of $\bar{H}$ with a well-defined initial location $z_{0}$ above the mirror:

$\Psi(z, t=0)=\sqrt{\frac{1}{\sigma \sqrt{\pi}}} \exp \left(-\frac{\left(z-z_{0}\right)^{2}}{2 \sigma^{2}}\right)$,

$\sigma$ is the spatial size of the initial state.

We study the vertical motion and ignore the classical radial motion in the following.

Evolution of the wave-function $\Psi(z, t)$ is given by the following expression:

$\Psi(z, t)=\int \Psi\left(z^{\prime}, 0\right) K\left(z^{\prime}, z, t\right) d z^{\prime}$.

Here $K\left(z,,^{\prime} z, t\right)$ is the particle propagator in GQSs:

$K\left(z,{ }^{\prime} z, t\right)=\sum_{i} \frac{\operatorname{Ai}\left(-\lambda_{i}\right)}{\left|\operatorname{Ai}^{\prime}\left(-\lambda_{i}\right)\right|^{2}} \operatorname{Ai}\left(\xi_{i}\right) \exp \left(-i \lambda_{i} \omega_{g} t\right)$.

$\omega_{g}$ is a characteristic gravitational frequency:

$\omega_{g}=\sqrt[3]{\frac{m g^{2}}{2 \hbar}}=903.362 s^{-1}$

$\lambda_{i}$ is an eigenvalue (complex) of GQS, $\xi_{i}=z / l_{g}-\lambda_{i}$.

In the limit $\sigma \ll l_{g}$, the following simplified expression for the wave function is valid:

$\Psi(z, t)=\frac{\sqrt{2 \sigma \sqrt{\pi}}}{l_{g}} \sum_{i} \frac{\operatorname{Ai}\left(\xi_{i}^{0}\right)}{\left|\operatorname{Ai}^{\prime}\left(-\lambda_{i}\right)\right|^{2}} \operatorname{Ai}\left(\xi_{i}\right) \exp \left(-i \lambda_{i} \omega_{g} t\right)$.

Here $\xi_{i}^{0}=z_{0} / l_{g}-\lambda_{i}$.

Due to the weak annihilation of $\bar{H}$ on the surface, the mirror plays the role of a detector. The corresponding rate of disappearance of $\bar{H}$ is given by the following expression 
[41]:

$$
\begin{aligned}
d P / d t & =-\frac{2 \sigma \sqrt{\pi}}{l_{g}} \frac{\Gamma}{\hbar} \exp (-\Gamma t / \hbar) S . \\
S & =\sum_{i, j}\left(T_{i j} \delta_{i j}+2 \operatorname{Re} T_{i j}\right) . \\
T_{i j} & =(-1)^{i+j} \frac{\operatorname{Ai}\left(\xi_{i}^{0}\right) \operatorname{Ai}\left(\xi_{j}^{0}\right)}{\left|\operatorname{Ai}^{\prime}\left(-\lambda_{i}\right) \operatorname{Ai}^{\prime}\left(-\lambda_{j}\right)\right|} \exp \left(-i \omega_{i j} t\right) .
\end{aligned}
$$

The interference terms in expression (28) are controlled by the frequencies $\omega_{i j}$ of transitions between GQSs. Measurements of the transition frequencies give access to the characteristic gravitational energy value $\varepsilon_{g}(5)$.

\subsection{Measuring the momentum distribution in GQSs}

\subsubsection{Sudden mirror drop}

The vertical momentum (velocity) distribution $F(p)$ provides information about spatial properties of GQSs. A method to measure it for $\bar{H}$ can consist in a prompt downward shift of the mirror. The mirror acceleration $a$ should significantly exceed the free fall acceleration $g$. The vertical shift should significantly exceed the characteristic gravitational length scale $l_{g}(5)$ and can be achieved using a piezoactuator. Then, the initial superposition of GQSs starts falling freely at time $t_{0}$ down to the mirror (detector) installed at a distance $H_{d}$ below. One can use the sudden approximation to describe this motion. A fraction of the $\bar{H}$ annihilate; the remaining $\bar{H}$ are reflected from the surface of the mirror due to quantum reflection and continue bouncing until their full annihilation.

The initial momentum distribution $F(p)$ is mapped into the time distribution of free fall events $[45,46]$ :

$$
\Phi \simeq m g\left|F\left(m g\left(t-t_{f}\right)\right)\right|^{2} .
$$

Here $\Phi$ is the flux of $\bar{H}$ falling on the annihilation surface, $t_{f}=\sqrt{2 H_{d} / g}$ is the classical free fall time.

The momentum distribution $F\left(p, t_{0}\right)\left(t_{0}\right.$ is the moment of the sudden mirror drop) can be evaluated by Fourier transform of the distribution (27) taken at time $t_{0}$. By measuring the time distribution of free fall events, one obtains the momentum distribution of GQS, which gives access to the characteristic spatial scale of GQSs $l_{g}(5)$.

\subsubsection{Prompt kick}

One could also use a prompt kick to make all $\bar{H}$ acquire the same upward momentum $p_{0}$. Such a kick could be achieved either by absorbing a photon from a laser beam, or by prompt switching of a gradient magnetic field $W(t, z)=$ $f\left(t-t_{0}\right) \mu z \partial B / \partial z \cdot f\left(t-t_{0}\right)$ is a function, which characterizes the time dependence of the gradient magnetic field and is localized around $t_{0}$ with a typical dispersion $\tau \ll 1 / \omega_{g}$. In the limit $\tau \rightarrow 0$, the wave-function change after promptly switching the field is:

$\Psi\left(z, t_{0}^{+}\right)=\exp \left(i p_{0} z\right) \Psi\left(z, t_{0}^{-}\right)$.

Here $t_{0}^{+}$and $t_{0}^{-}$are the moments just after and before the kick, and

$p_{0}=\mu \partial B / \partial z \int f\left(t-t_{0}\right) d t$.

The momentum distribution in an upstream detector is:

$P \simeq m g\left|F\left(m g\left(t-t_{f}\right)+p_{0}\right)\right|^{2}$.

Evaluating the energy and spatial gravitational scales from interferometry experiments and comparing these values with theory, one can conclude on a presence of extra interactions between atom and mirror at a micrometer scale.

\section{Conclusion}

We have proposed in this paper a new method for producing long confinement times in gravitational quantum states (GQSs) and a magneto-gravitational trap (MGT) for atoms, anti-atoms, neutrons and other particles possessing a magnetic moment. The Earth gravitational field and a reflecting mirror confine the particles in the vertical direction. The interaction of the particle's magnetic moment and the magnetic field originating from an electric current passing through a vertically installed wire confines particles in the radial direction, combined with the centrifugal acceleration of the particles. We underline that the observation time is the key parameter that defines the precision of measurements of such states. In case of anti-hydrogen atoms, one can achieve the observation time limited only by their annihilations in the surface [75]. In case of hydrogen atoms, storage times will be significantly longer due to a contribution from their specular reflection from the surface in the case of direct contact. In case of neutrons, storage times can approach the neutron lifetime. Our analysis shows that the mixing of vertical and horizontal motions of particles can be controlled to an acceptable level not prohibiting precision measurements of GQSs in the MGT. We give examples, which prove the feasibility of precision studies of GQS in the MGT.

In the limit of low particle velocities and magnetic fields, precise control of the particle motion and long storage times in the MGT can provide ideal conditions for gravitational spectroscopy: for the sensitive verification of the equivalence 
principle for $\bar{H}$; for improving constraints on extra fundamental interactions from experiments with $n$, atoms and $\bar{H}$. These ideas can be applied in particular to the GRASIAN (co-authors of the present article), GBAR [72] and GRANIT [73] projects. S.V. thanks Academy of Finland for support (Grant N.317141).

Data Availability Statement This manuscript has no associated data or the data will not be deposited. [Authors' comment: This work proposes and develops a new method and does not contain experimental data.]

Open Access This article is licensed under a Creative Commons Attribution 4.0 International License, which permits use, sharing, adaptation, distribution and reproduction in any medium or format, as long as you give appropriate credit to the original author(s) and the source, provide a link to the Creative Commons licence, and indicate if changes were made. The images or other third party material in this article are included in the article's Creative Commons licence, unless indicated otherwise in a credit line to the material. If material is not included in the article's Creative Commons licence and your intended use is not permitted by statutory regulation or exceeds the permitted use, you will need to obtain permission directly from the copyright holder. To view a copy of this licence, visit http://creativecomm ons.org/licenses/by/4.0/.

Funded by SCOAP ${ }^{3}$.

\section{References}

1. V.I. Luschikov, A.I. Frank, JETP Lett. 28, 559 (1978)

2. V.V. Nesvizhevsky, H.G. Boerner, A.M. Gagarski, G.A. Petrov, A.K. Petukhov, H. Abele, S. Baessler, T. Stoferle, S.M. Soloviev, Nucl. Instrum. Methods A 440, 754 (2000)

3. V.V. Nesvizhevsky, H.G. Boerner, A.K. Petukhov, H. Abele, S. Baessler, F.J. Ruess, T. Stoferle, A. Westphal, A.M. Gagarski, G.A. Petrov, A.V. Strelkov, Nature 415, 297 (2002)

4. V.V. Nesvizhevsky, H.G. Boerner, A.M. Gagarski, A.K. Petukhov, G.A. Petrov, H. Abele, S. Baessler, G. Divcovic, F.J. Ruess, T. Stoferle, A. Westphal, A.V. Strelkov, K.V. Protasov, AYu. Voronin, Phys. Rev. D 67, 102002 (2003)

5. V.V. Nesvizhevsky, A.K. Petukhov, H.G. Boerner, K.V. Protasov, AYu. Voronin, A. Westphal, S. Baessler, H. Abele, A.M. Gagarski, Phys. Rev. D 68, 108702 (2003)

6. V.V. Nesvizhevsky, A.K. Petukhov, H.G. Boerner, T.A. Baranova, A.M. Gagarski, G.A. Petrov, K.V. Protasov, AYu. Voronin, S. Baessler, H. Abele, A. Westphal, L. Lucovac, Eur. Phys. J. C 40, 479 (2005)

7. A. Westphal, H. Abele, S. Baessler, V.V. Nesvizhevsky, K.V. Protasov, AYu. Voronin, Eur. Phys. J. C 51, 367 (2007)

8. R.W. Robinett, Phys. Rep. 392, 1 (2004)

9. M. Berberan-Santos, E. Bodunov, L. Pogliani, J. Math. Chem. 37, $101(2005)$

10. W.H. Mather, R.F. Fox, Phys. Rev. A 73, 032109 (2006)

11. O. Bertolami, J.G. Rosa, Phys. Lett. B 633, 111 (2006)

12. E. Romera, F. de los Santos, Phys. Rev. Lett. 99, 263601 (2007)

13. G. Della Valle, M. Savoini, M. Ornigotti, P. Laporta, V. Foglietti, M. Finazzi, L. Duo, S. Longhi, Phys. Rev. Lett. 102, 180402 (2009)

14. I.L. Garanovich, S. Longhi, A.A. Sukhorukov, Y.S. Kivshar, Phys. Rep. 518, 1 (2012)

15. M. Belloni, R.W. Robinett, Phys. Rep. 540, 25 (2014)

16. H. Abele, S. Baessler, A. Westphal, Lect. Notes Phys. 631, 355 (2003)
17. A. Frank, P. van Isacker, J. Gomez-Camacho, Phys. Lett. B 582, 15 (2004)

18. F. Brau, F. Buisseret, Phys. Rev. D 74, 036002 (2006)

19. F. Buisseret, B. Silvestre-Brac, M. Vincent, Class. Quantum Grav. 24, 855 (2007)

20. S. Baessler, V.V. Nesvizhevsky, K.V. Protasov, AYu. Voronin, Phys. Rev. D 75, 075006 (2007)

21. I. Antoniadis, S. Baessler, M. Buchner, V.V. Fedorov, S. Hoedl, A. Lambrecht, V.V. Nesvizhevsky, G. Pignol, K.V. Protasov, S. Reynaud, Yu. Sobolev, Compt. Rend. Phys. 12, 755 (2011)

22. P. Brax, G. Pignol, Phys. Rev. Lett. 107, 111301 (2011)

23. O. Bertolami, F.M. Nunes, Class. Quantum Grav. 20, L61 (2003)

24. C. Kiefer, C. Weber, Ann. Phys. 14, 253 (2005)

25. E. Kajari, N.L. Harshman, E.M. Rasel, S. Stenholm, G. Sussmann, W.P. Scheich, Appl. Phys. B 100, 43 (2010)

26. O. Bertolami, J.G. Rosa, C.M.L. de Aragao, P. Castorina, D. Zappala, Phys. Rev. D 72, 025010 (2005)

27. A. Saha, Eur. Phys. J. C 51, 199 (2007)

28. K. Nozari, P. Pedram, Eur. Phys. Lett. 92, 50013 (2010)

29. P. Pedram, K. Nozari, S.H. Taheri, J. HEP 3, 093 (2011)

30. A. Kobakhidze, Phys. Rev. D 83, 021502 (2011)

31. M. Chaichian, M. Oksanen, A. Tureanu, Phys. Lett. B 702, 419 (2011)

32. A. Martin-Ruiz, C.A. Escobar, Phys. Rev. D 97, 095039 (2018)

33. C.A. Escobar, A. Martin-Ruiz, Phys. Rev. D 99, 075032 (2019)

34. A.N. Ivanov, M. Wellenzohn, H. Abele, Phys. Lett. B 797, 134819 (2019)

35. V.V. Nesvizhevsky, K.V. Protasov, in: Trends in Quantum Gravity Research, Editor: David C. Moore, pp. 65-107, (2006)

36. V.V. Nesvizhevsky, Phys. Uspekhi 53, 645 (2010)

37. T. Jenke, P. Geltenbort, H. Lemmel, H. Abele, Nat. Phys. 7, 468 (2011)

38. T. Jenke, G. Gronenberg, J. Burgdorfer, L.A. Chizhova, P. Geltenbort, A.N. Ivanov, T. Lauer, T. Lins, S. Rotter, H. Saul, U. Schmidt, H. Abele, Phys. Rev. Lett. 112, 151105 (2014)

39. G. Ichikawa, S. Komamiya, Y. Kamiya, Y. Minami, M. Tani, P. Geltenbort, K. Yamamura, M. Nagano, T. Sanuki, S. Kawasaki, M. Hino, M. Kitaguchi, Phys. Rev. Lett. 112, 071101 (2014)

40. V.V. Nesvizhevsky, AYu. Voronin, R. Cubitt, K.V. Protasov, Nat. Phys. 6, 114 (2010)

41. AYu. Voronin, P. Froelich, V.V. Nesvizhevsky, Phys. Rev. A 83, 032903 (2011)

42. G. Dufour, P. Debu, A. Lambrecht, V.V. Nesvizhevsky, S. Reynaud, AYu. Voronin, Eur. J. Phys. C 74, 2371 (2014)

43. AYu. Voronin, V.V. Nesvizhevsky, O.D. Dalkarov, E.A. Kupriyanova, P. Froelich, Hyp. Inter. 228, 133 (2014)

44. A.Y. Voronin, V.V. Nesvizhevsky, G. Dufour, S. Reynaud, J. Phys. B 49, 054001 (2016)

45. V.V. Nesvizhevsky, A.Y. Voronin, P.P. Crepin, S. Reynaud, Hyp. Inter. 240, 32 (2019)

46. P.P. Crepin, C. Christen, R. Guerout, V.V. Nesvizhevsky, AYu. Voronin, S. Reynaud, Phys. Rev. A 99, 042119 (2019)

47. S. Vasiliev, J. Ahokas, J. Jarvinen, V.V. Nesvizhevsky, A.Y. Voronin, F. Nez, S. Reynaud, Hyp. Inter. 240, 14 (2019)

48. P. Crivelli, V.V. Nesvizhevsky, A.Y. Voronin, Adv. HEP 2015, $173572(2015)$

49. J. Schmiedmayer, Phys. Rev. A 52, R13 (1995)

50. J. Denschlag, D. Cassettari, A. Chenet, S. Schneider, J. Schmiedmayer, Appl. Phys. B 69, 291 (1999)

51. J. Denschlag, D. Cassettari, J. Schmiedmayer, Phys. Rev. Lett. 82, 2014 (1999)

52. M. Daum, P. Fierlinger, B. Franke, P. Geltenbort, L. Goeltl, E. Gutsmiedl, J. Karch, G. Kessler, K. Kirch, H.-C. Koch, A. Kraft, T. Lauer, B. Lauss, E. Pierre, G. Pignol, D. Regianni, P. SchmidtWellenburg, Yu. Sobolev, T. Zechlau, G. Zigmond, Phys. Lett. B 704, 456 (2011) 
53. T.H. Brenner, S. Chesnevskaya, P. Fierlinger, P. Geltenbort, E. Gutsmiedl, T. Lauer, K. Rezai, J. Rothe, T. Zechlau, R. Zoud, Phys. Lett. B 741, 316 (2015)

54. E.L. Raab, M. Prenties, A. Cable, S. Chu, D.E. Pritchard, Phys. Rev. Lett. 59, 2631 (1987)

55. K.B. Davis, M.O. Mewes, M.R. Andrews, N.J. van Druten, D.S. Durfee, D.M. Kim, W. Ketterle, Phys. Rev. Lett. 75, 3969 (1995)

56. M.H. Andersen, J.R. Ensher, M.R. Matthews, C.E. Wieman, E.A. Cornell, Science 269, 198 (1995)

57. G. Pignol, S. Baessler, V.V. Nesvizhevsky, K. Protasov, D. Rebreyend, A.Y. Voronin, Adv. HEP 2014, 628125 (2014)

58. P.-P. Crepin, G. Dufour, R. Guerout, A. Lambrecht, S. Reynaud, Phys. Rev. A 95, 032501 (2017)

59. S. Baessler, V.V. Nesvizhevsky, G. Pignol, K.V. Protasov, D. Rebreyend, E.A. Kupriyanova, A.Y. Voronin, Phys. Rev. D 91, 042006 (2015)

60. S. Baessler, V.V. Nesvizhevsky, G. Pignol, K.V. Protasov, D. Rebreyend, E.A. Kupriyanova, A.Y. Voronin, Phys. Rev. D 91, 069905 (2015)

61. A.E. Meyerovich, V.V. Nesvizhevsky, Phys. Rev. A 73, 063616 (2006)

62. A.Y. Voronin, H. Abele, S. Baessler, V.V. Nesvizhevsky, A.K. Petukhov, K.V. Protasov, Westphal Phys. Rev. A 73, 044029 (2006)

63. A.Y. Voronin, E.A. Kupriyanova, A. Lambrecht, V.V. Nesvizhevsky, S. Reynaud, J. Phys. B 49, 205003 (2016)

64. C. Codau, V.V. Nesvizhevsky, M. Fertle, G. Pignol, K.V. Protasov, Nucl. Instrum. Methods A 677, 10 (2012)

65. R. Golub, J.M. Pendlebury, Phys. Lett. A 53, 133 (1975)

66. G.P. Pron'ko, Y.G. Stroganov, JETP 72, 2048 (1977)

67. R. Blumel, K. Dietrich, Phys. Lett. A 139, 236 (1989)

68. P.P. Crepin, E.A. Kupriyanova, R. Guerout, A. Lambrecht, V.V. Nesvizhevsky, S. Reynaud, S. Vasiliev, A.Y. Voronin, Eur. Phys. Lett. 119, 33001 (2017)
69. I.I. Rabi, S. Millman, P. Kusch, J.R. Zacharias, Phys. Rev. 55, 526 (1939)

70. V.I. Luschikov, Y.N. Pokotilovsky, A.V. Strelkov, F.L. Shapiro, JETP Lett. 9, 23 (1969)

71. A. Steyerl, Phys. Lett. B 29B, 33 (1969)

72. P. Indelicato, G. Chardin, P. Grandemange, D. Lunney, V. Manea, A. Badertscher, P. Crivelli, A. Curioni, A. Marchionni, B. Rossi, A. Rubbia, V.V. Nesvizhevsky, D. Brook-Roberge, P. Comini, P. Debu, P. Dupre, L. Liszkay, B. Mansoulie, P. Perez, Hyprf. Inter. 228, 141 (2014)

73. D. Roulier, F. Vezzu, S. Baessler, B. Clement, D. Morton, V.V. Nesvizhevsky, G. Pignol, D. Rebreyend, Adv. High En. Phys. 2015, 730437 (2015)

74. M. Escobar, F. Lamy, A.E. Meyerovich, V.V. Nesvizhevsky, Adv. High En. Phys. 2014, 764182 (2014)

75. P.P. Crepin, E.A. Kupriyanova, R. Guerout, A. Lambrecht, V.V. Nesvizhevsky, S. Reynaud, S. Vasiliev, A.Y. Voronin, Hyperf. Int. 240, 58 (2019)

76. H. Abele, D. Dubbers, H. Hase, M. Klein, A. Knopfler, M. Kreuz, T. Lauer, B. Markisch, D. Mund, V.V. Nesvizhevsky, A. Petoukhov, C. Schmidt, M. Schumamm, T. Soldner, Nucl. Instrum. Methods A 562, 407 (2006)

77. P. Schmidt-Wellenburg, K.H. Andersen, P. Coutois, M. Kreuz, S. Mironov, V.V. Nesvizhevsky, G. Pignol, K.V. Protasov, T. Soldner, F. Vezzu, O. Zimmer, Nucl. Instrum. Methods A 611, 267 (2009)

78. G. Cronenberg, Ph Brax, H. Filter, P. Geltenbort, T. Jenke, G. Pignol, M. Pitschumann, M. Thalhammer, H. Abele, Nat. Phys. 14, 1022 (2018)

79. C.C. Silvera, I.F. Stoof, H.T.C. Verhaar, Phys. Rev. Lett. 62, 2361 (1989)

80. E. Fermi, Ric. Sci. 7, 13 (1936) 